\title{
Smart Composite Silica Nanomaterials
}

\author{
Ignác Capek* \\ Slovak Academy of Sciences, Institute of Measurement Sciences, Slovakia
}

*Corresponding author: Ignác Capek, Slovak Academy of Sciences, Institute of Measurement Sciences, Slovakia

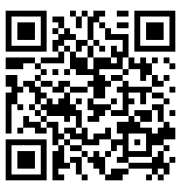

\section{ARTICLE INFO}

Received: 幽 November 26, 2019

Published: 仹 December 02, 2019

Citation: Chris Caulkins. Detection of Psychological Trauma and Suicide Risk among Emergency Medical Services Personnel an Artificial Intelligence Approach. Biomed J Sci \& Tech Res 23(3)-2019. BJSTR. MS.ID.003893.

\section{ABSTRACT}

The composite silica-based nanoparticles are prepared by various synthetic approaches. There are characterized by an enormous large particle surface. This favors the particle surface interaction with functional polymers and drugs. The data show that the conjugation of high molecule weight functional (co)polymers with the particle surface improves the particle stability and bioavailability. This increases the loading capacity of porous silica nanoparticles and the efficiency of drug delivery systems.

Keywords: Silica Nanoparticles; Particle Size; Drugs; Loading Capacity

Abbreviations: PNPs: Polymer Nanoparticles; PSiNPs: Porous Silica Nanoparticles; DDS: Drug Delivery Systems; PEG: Polyethylene Glycol; PMVA-MA: Poly Methyl Vinyl Ether-Alt-Maleic Acid; DXR: Doxorubicin; PTX: Paclitaxel; MTX: Methotrexate

\section{Mini Review}

The preparation of polymer nanoparticles (PNPs) is devoted to the synthesis of composite nanomaterials with requested design and structure. This reaction mechanism allow to syntheses nanoparticles with low toxicity and high stability. Although the potential of bare porous silica nanoparticles (PSiNPs) has already been shown in the drug delivery systems (DDS) [1], the functionalized particle surface to improve the biological and physicochemical properties of the nanoparticles. The modified PNPs were observed as efficient DDSs. The presence of covalently-bound polymer(s) or functional groups on the PSiNPs surface not only improved the hydrophilicity/hydrophobicity of the particles, but also played a crucial role in augmenting the aqueous dispersibility of the NPs as a result of the electrostatic or steric repulsion forces [2] and thus, preventing the PSiNP's aggregation. The presence of charged polymers and functional groups increase the zeta potential of NPs. This is connected with a futher improvement of the NPs stability due to the enhanced repulsion between nanoparticles [3]. The above-mentioned synthetic processes allow to prepare particles ranging in the size from $10 \mathrm{~nm}$ to several $\mu \mathrm{ms}$. The microemulsion approach, for example, holds the nanoparticle below $50 \mathrm{~nm}$.

Stober et al.' sol-gel widely explored process holds the preparation of monodisperse solid inorganic particles ranging in size $50 \mathrm{~nm}$ to $2 \mu \mathrm{m}$ [4]. The researchers have widely used this approach for the synthesis of various bare, porous and functionalized inorganic and composite particles. The porous silica nanoparticles were prepared by the reverse microemulsion process as reported by Lin and co-workers [5]. This process allowed to prepare the monodisperse dispersion compared with other approaches. PSiNPs were successfully also prepared by the evaporation-induced selfassembly process. The evaporation of alcohol induces the increased concentration of surfactants and their self-assembly into micelles. This process holds the formation of liquid-crystal mesophasee [6]. It generates the core/shell structures with various cores and shells. The coating (shell) holds desired type and concentration of polymers and functional groups [7]. The type and concentration of additives (agent precursors) are responsible for the formed particle morphology [8]. In the case of PSiNPs the apperance of cargoes is the result of simple incorporation of agents into the pores. The proceeds by physical adsorption and/or by covalent bonding of additives with the surface groups.

The particle size and morphology can be effectively controlled by the concentration and type of additives surfactants and cosurfactants such as alcohols, amines, inorganic salts and organic compounds of amphiphilic properties. This is also the case with some tetra alkoxysilanes with different alkoxy groups. The addition of alcohols can vary the particle size due to the change in the 
solvent power. A widely explored additive poly(ethylene glycole) (PEG) is used to modify the various particles including silica NPs. The adsorbed PEG copolymers or PEG modified particles contribute to the stabilization of particles by the steric effect. Among the PEG-based amphiphilic copolymers belong the triblock copolymer Pluronic F127 broadly used to prepare and stabilize silica dispersion with the average particle size ca. $300 \mathrm{~nm}$ [9]. Therapeutic and drug cargoes in particle matrix is based on the adsorption properties of PSiNPs. The PSiNPs' pores are able to adsorb both hydrophilic and hydrophobic additives (drugs). The large number of pores creates the large pore volume and so the large loading capacity. Furthermore, their capacity can be further increased by utilizing polymer gate keeping for the entrapment of hydrophobic therapeutics [10].

The presence of functional particle surface groups induces their intermolecular interaction with drugs and favor the consecutive drug loading steps [11]. This loading drug mechanisms depends also on the concentration of agents or the drug feeding ratio [12]. The most important parameter of the drug loading steps is the pore volume of silica nanoparticles. The second main step of DDS is the release profile of drugs from PSiNPs. The drug release is controlled by its diffusion from the pores. This process depends on the type and amount of functional groups within the pores as well as the type of drugs. Thus, the drug release results from the intermolecular or group interactions between the discussed entities [13]. The particle surface and pores modified, for example, with functional polymers such as cationic polyethylene or anionic poly(methyl vinyl ether-alt-maleic acid) (PMVE-MA) copolymer improve the colloidal stability and bio adhesive properties of PSiNPs [14]. In line with these results, there have been evidences of high uptake of negatively charged particles in different cell lines [15], despite the unfavorable interaction between them and the negatively charged cell membranes [16]. The uptake of drugs/ therapeutics is controlled by their interaction with the functional groups of surface-adsorbed polymers or copolymers.

The presence of functional polymers adsorbed on the silica particle surface increases the amount of drugs such as doxorubicin (DXR), paclitaxel (PTX), methotrexate (MTX), etc. For example, the amount of MTX on the bare PSiNPs reaches the value $~ 6.4 \%$. On the contrary, the particles modified with PEI and PMVE-MA are characterized with the drug loading degree $\sim 12.6$ and $14.0 \%$, respectively [17]. The drug-loaded particles are accumulated into tumor via enhanced permeability and retention effect [18]. This is because tumor vasculature is generally more leaky compared to normal vasculature due to rapid growth and thus nanoscale materials can be passively extravagated into the tumor interstitial. However, the lymphatic drainage system of the tumor tissue is impaired and thus the nanoparticles cannot be excluded as wastes. So, the therapeutic-loaded nanoparticles are trapped in the tumor during their blood circulation. The drug release from nanoparticles is controlled by its diffusion and it saturates the tumor tissue with therapeutics. As reported, all tumors treated with both nanoparticles and laser underwent complete necrosis by day 10 without regrowth over 90 days.

\section{Conclusion}

The porous silica-based nanoparticles are prepared by various synthetic approaches. There are characterized by an enormous large particle surface. This favor the particle surface interaction with functional polymers and later with drugs. The previous data show that the conjugation of low molecular and high molecule weight functional (co) polymers with the particle surface improves the particle stability and bioavailability. Such nanoparticles are able to intermolecular interact with drugs/therapeutics. This increases the loading capacity of PSiNPs and the efficiency of DDSs. The bare PSiNPs can be decorate with various polymers and copolymers. Their functionalization leads to efficient loading of drugs as a result of loading of cargoes into the particle cores.

\section{References}

1. Shahbazi MA, Hamidi M, Mäkilä EM, Zhang H, Almeida PV, et al. (2013) The mechanisms of surface chemistry effects of mesoporous silicon nanoparticles on immunotoxicity and biocompatibility. Biomaterials 34(31): 7776-7789

2. Ayala V, Herrera AP, Latorre Esteves M, Torres Lugo M, Rinaldi C (2013) Effect of surface charge on the colloidal stability and in vitro uptake of carboxymethyl dextran-coated iron oxide nanoparticles. J Nanopart Res 15(8): 1874.

3. Shahbazi MA, Hamidi M, Mohammadi Samani S (2013) Preparation, optimization, and in-vitro/in-vivo/ex-vivo characterization of chitosanheparin nanoparticles: drug-induced gelation. J Pharm Pharmacol 65(8): 1118-1133.

4. Stober W, Fink A, Bohn E (1968) Controlled growth of monodisperse silica spheres in the micron size range. J Colloid Interface Sci 26(1): 6269.

5. Lin YS, Wu SH, Tseng CT, Hung Y, Chang C, et al. (2009) Synthesis of hollow silica nanospheres with a microemulsion as the template. Chem Commun 24: 3542-3544.

6. Brinker CJ, Lu Y, Sellinger A, Fan H (1999) Evaporation-induced selfassembly: Nanostructures made easy. Adv Mater 11(7): 579-585.

7. Lou XW, Archer LA, Yang Z (2008) Hollow Micro-/nanostructures: Synthesis and applications. Adv Mater 20(21): 3987-4019.

8. Trewyn BG, Slowing II, Giri S, Chen HT, Lin VSY (2007) Synthesis and functionalization of a mesoporous silica nanoparticle based on the solgel process and applications in controlled release. Acc Chem Res 40(9): 846-853.

9. Wanyika H, Gatebe E, Kioni P, Tang Z, Gao Y (2011) Synthesis and characterization of ordered mesoporous silica nanoparticles with tunable physical properties by varying molar composition of reagents. Afr J Pharm Pharmacol 5(21): 2402-2410.

10. Palanikumar L, Kim HY, Oh JY, Thomas AP, Choi ES, et al. (2015) Noncovalent surface locking of mesoporous silica nanoparticles for exceptionally high hydrophobic drug loading and enhanced colloidal stability. Biomacromolecules 16(9): 2701-2714.

11. Vallet Regí M, Balas F, Arcos D (2007) Mesoporous materials for drug delivery. Angew Chem Int Ed 46(40): 7548-7558.

12. Zhu Y, Shi J, Shen W, Chen H, Dong X, et al. (2005) Preparation of novel hollow mesoporous silica spheres and their sustained-release property. Nanotechnology 16: 2633. 
13. Nieto A, Colilla M, Balas F, Vallet Regí M (2010) Surface electrochemistry of mesoporous silicas as a key factor in the design of tailored delivery devices. Langmuir 26(7): 5038-5049.

14. Shahbazi MA, Almeida PV, Mäkilä E, Correia A, Ferreira MP, et al. (2014) Poly(methyl vinyl ether-alt-maleic acid)-functionalized porous silicon nanoparticles for enhanced stability and cellular internalization. Macromol Rapid Commun 35(6): 624-629.

15. Villanueva A, Canete M, Roca AG, Calero M, Veintemillas Verdaguer $S$, et al. (2009) The influence of surface functionalization on the enhanced internalization of magnetic nanoparticles in cancer cells. Nanotechnology 20: 115103.

\section{ISSN: 2574-1241}

DOI: 10.26717/BJSTR.2019.23.003894

Ignác Capek. Biomed J Sci \& Tech Res

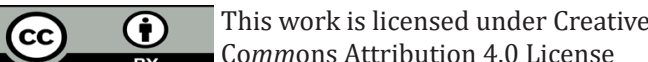

Submission Link: https://biomedres.us/submit-manuscript.php
16. Wang D, Huang J, Wang X, Yu Y, Zhang H, et al. (2013) The eradication of breast cancer cells and stem cells by 8-hydroxyquinoline-loaded hyaluronan modified mesoporous silica nanoparticle-supported lipid bilayers containing docetaxel. Biomaterials 34(31): 7662-7673.

17. Shahbazi MA, Almeida PV, Mäkilä EM, Kaasalainen MH, Salonen JJ, et al. (2014) Biomaterials 35: 7488-7500.

18. Uchegbu IF (2006) Pharmaceutical nanotechnology: polymeric vesicles for drug and gene delivery. Expert Opin Drug Del 3(5): 629-640.

BIOMEDICAL
RESEARCHES $\quad \begin{aligned} & \text { Assets of Publishing with us } \\ & \text { - Global archiving of articles }\end{aligned}$

